\title{
Feedback from the European Bioanalysis Forum liquid microsampling consortium: capillary liquid microsampling and assessment of homogeneity of the resultant samples
}

Zoe Cobb1, Morten Rohde², lain Love ${ }^{3}, V^{1}$ alerie Boutet ${ }^{4}$, Katrin Schroeter $^{5}$, Glen

Hawthorne $^{6}$, Lieve Dillen 7 , Matthew Barfield ${ }^{8}$, Abdullah Kandira $^{5}$, Marion Kranenborgh ${ }^{9}$, Stephen White ${ }^{8}$ \& Philip Timmerman*,10

${ }^{1}$ LGC, Newmarket, England, UK

${ }^{2}$ Lundbeck, Valby, Denmark

${ }^{3}$ Charles River, Edinburgh, UK

${ }^{4}$ Sanofi, Paris, France

${ }^{5}$ Sanofi, Frankfurt, Germany

${ }^{6}$ AstraZeneca, Cambridge, UK

${ }^{7}$ Janssen R\&D, Beerse, Belgium

${ }^{8}$ GlaxoSmithKline, Ware, England, UK

${ }^{9} \mathrm{QPS}$, Groningen, The Netherlands

${ }^{10}$ European Bioanalysis Forum, Havenlaan 86c b204, 1000 Brussels, Belgium

*Author for correspondence: chair@e-b-f.eu

Following the completion of a detailed experimental protocol into the potential inhomogeneity of capillary liquid microsamples, which was performed at seven European Bioanalysis Forum member companies, the summary and conclusion on the data are reported here. It has been demonstrated that it is possible to generate homogeneous samples using these microsampling techniques; that the resultant microsamples can be accurate and precise and that capillary liquid microsampling data can be consistent with conventional larger volume plasma samples. However, the data contain some variability which is contributed to by the different range of experiences that each investigating site had with these techniques. Therefore, knowledge of the compounds, well-designed experiments and experience with these techniques are essential for the delivery of high quality data.

First draft submitted: 2 January 2019; Accepted for publication: 14 February 2019; Published online: 11 April 2019

Keywords: capillary $\bullet \mathrm{EBF} \bullet$ homogeneity $\bullet$ microsampling

Within the bioanalytical community, there is significant interest in the drive toward microsampling for the collection of matrix samples owing to potential ethical concerns and scientific benefits. To support this drive, it is important to ensure that the quantitative data derived remain of the highest quality. Implementation of microsampling requires scaling down sample volumes and alternative approaches to the production and storage of plasma samples.

For a significant period of time the European Bioanalysis Forum (EBF) has been engaging its members in high level technical discussions on the use and implementation on microsampling techniques. Initially, this focused on dried blood spots (DBS) [1] and more recently has refocused on liquid microsampling (LMS). In 2014, the EBF-DBS microsampling consortium was rebranded to the EBF-LMS consortium [2]. This group has since spent a period of time reflecting on the considerations for implementing LMS techniques [3], as well as designing, conducting and analyzing the results from experimental protocols.

Two areas of investigation were addressed by the experimental protocols:

- Technical challenges in handling of small volumes;

- Perceived concerns of inhomogeneity relating to small volumes. 


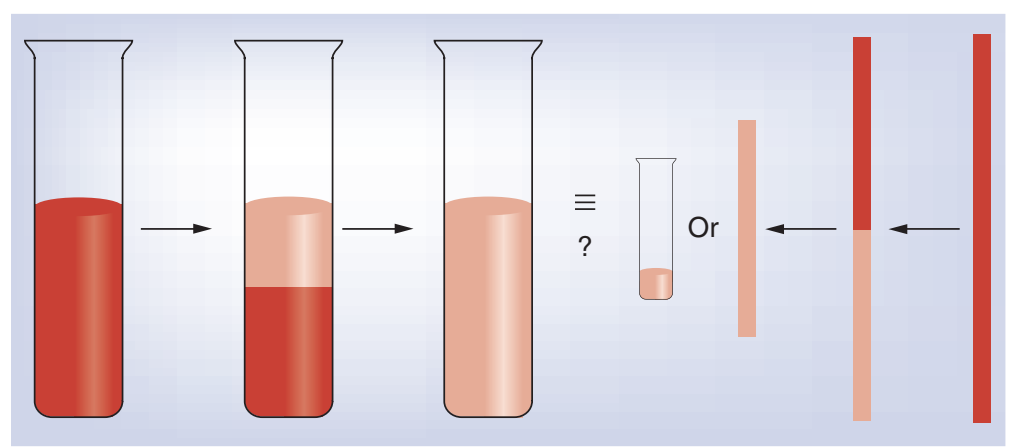

Figure 1. Are plasma samples generated by conventional and capillary techniques the same?

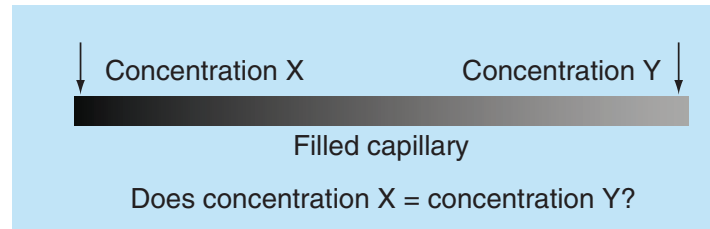

An experimental protocol to investigate the concerns identified in point 1 are detailed in a separate paper by Hawthorne et al. [4]. This paper reports on the results of an experimental protocol that was designed by the EBF-LMS consortium to address the perceived concerns of inhomogeneity in microsamples as highlighted in point 2. Within the protocol was an assessment of data obtained from a microsampling design using two common approaches (Vitrex ${ }^{\circledR}$ micro hematocrit tube and Drummond plasma capillaries) and a comparison of each against control samples prepared using conventional volumes and processes (see Figure 1).

One of the key questions the EBF-LMS consortium intended to address when the experimental protocols were prepared was 'are capillary microsamples any more or less susceptible to problems with homogeneity than traditional plasma samples?' as illustrated in Figure 2.

Sample homogeneity is a critical factor in reproducible analysis and the inability of the analyte to distribute freely within the entire bioanalytical sample was perceived to be a significant concern when DBS were being evaluated [5]. The same thoughts and considerations tend to arise when considering small liquid volumes. The consortium's experimental protocol was designed to identify if these concerns are justified and supported by experimental data.

The protocol prepared by the EBF-LMS consortium detailed a series of experiments aimed at investigating most of the variables perceived to affect sample homogeneity in LMS devices. The protocols were conducted by seven EBF member companies obtaining data for 15 different analytes, 14 of which were small molecules and one a monoclonal antibody. Each member company provided data for 1-3 analytes, and no analytes were measured at more than one site. The analytical end point of all of these assays was LC-MS/MS with the exception of the monoclonal antibody for which an ELISA was used.

The main experiments included in the protocol addressed the following questions:

- Are the results of liquid microsamples consistent with the respective conventional volume plasma samples?

- Does the volume of the sample tube selected for storage has any impact on the homogeneity of the samples?

- Is a plasma sample homogeneous within the capillary sampling device?

- If a dilution step is required to provide a viable sample at what point in the sampling or analytical process should this dilution be performed?

Two types of liquid microsampling devices were tested and the typical sample collection process for each is detailed below.

Vitrexcapillaries (Device 1):

- Blood is drawn up into a sampling capillary coated with anticoagulant (typically contains roughly $32 \mu \mathrm{l}$ but different sizes are available);

- A wax plug is inserted at one end and the capillary is placed in a tube for centrifugation; 
- Once centrifuged a ceramic cutter is used to cut the capillary at a point between the blood and plasma layers to produce one section of capillary containing plasma only;

- The plasma is transferred via capillary action to one or two 8 and/or $4 \mu \mathrm{l}$ accurate volume analytical capillaries;

- The capillaries are placed in a small tube for storage at -20 or $-80^{\circ} \mathrm{C}$;

- A dilution solvent, typically aqueous buffer containing protein is added to the tube (before or after freezing) and mixed to dilute the plasma sample and help draw the sample out of the capillary;

- This diluted sample is then aliquoted and taken through the appropriate extraction procedure.

Drumond device (Device 2):

- Blood is drawn up into a Drummond capillary (typically $70 \mu \mathrm{l}$ ) containing a thixotropic gel and coated with EDTA;

- The capillary is placed in a tube for centrifugation;

- During centrifugation, the thixotropic gel migrates to the interface between the plasma and corpuscular fraction;

- Once centrifuged all the plasma is dispensed into an appropriate tube using a reusable wiretrol ${ }^{\mathrm{TM}}$ plunger;

- An aliquot of this sample may then be taken for analysis using the appropriate extraction procedure;

- Where required an accurate volume of the plasma could be diluted with an appropriate dilution solvent (before or after freezing) to give a larger sample to handle prior to taking a subsample for analysis.

It is not the intention of the EBF to directly compare and/or promote one device over the other. These devices were, at the time of preparing the protocol, the two most prominently used techniques within the bioanalytical industry. Our intention was to take each device in isolation and address any perceived concerns relating to their use.

\section{Design of protocol}

Prior to performing the protocol, it was essential that each company participating in the work perform some preliminary work. The assays intended for use were all nonproprietary assays that could be used to support publication but many of these assays had not been developed for use with microsamples. The assays were therefore adapted to fit the lower sample volumes that would be used. This also allowed the contributing sites to build experience with both devices prior to executing the test protocol. During this phase, consideration was given to the selection of an appropriate dilution solvent to be used in the handling of microsamples.

Separate protocols were designed for the two devices as each device involved a different handling procedure as detailed above. These protocols were each performed alongside a fortified 'control' sample. The control was a blood sample of at least $1 \mathrm{ml}$ that was processed appropriately to yield a conventional volume plasma sample for comparison.

The protocol design detailed that analysis of $n=5 / 6$ were performed for all tests; however, it should be noted that for device $1, \mathrm{n}=6$ replicates was defined as $\mathrm{n}=6$ capillaries while for device $2, \mathrm{n}=6$ replicates were defined as $\mathrm{n}=6$ subsamples taken from a single microsample expelled into a sample tube from the microsampling device. As a result, a direct comparison of the two techniques from this dataset is not advised. In some instances, less than $\mathrm{n}=5$ replicates were obtained. $\mathrm{N}=3$ replicates or more were achieved for all investigations except the 4- $\mu \mathrm{l}$ Vitrex samples at quality control (QC) low of Diclofenac.

For each investigation, samples were prepared at concentrations equivalent to a $\mathrm{QC}$ low and QC high for each analyte in both control samples and microsamples. For all experiments performed the results from the microsamples are reported as a \%bias from the control sample. However, it should be noted that there was variability in the process used to prepare controls. In some cases, they were prepared from the same fortified sample used to generate the microsample. This gave a higher level of anticoagulant in the microsamples than the control owing to the presence of anticoagulant with the LMS device. In other cases, they were prepared from a separate fortified plasma sample.

\section{Results}

\section{Comparison of microsamples to traditional samples}

Tables 1 \& 2 show the tabulated precision data for devices 1 and 2 and the accuracy of the results from both devices compared with the relevant control samples. Table 1 shows the individual data for QC low and high for each analyte and Table 2 shows the percentage of results within a criteria of 15, 10 and 5\% or outside 15\%. 


\begin{tabular}{|c|c|c|c|c|c|c|c|c|c|c|c|}
\hline \multirow[t]{2}{*}{ Site } & \multirow[t]{2}{*}{ Compound } & \multirow[t]{2}{*}{ Concentration } & \multicolumn{2}{|c|}{$\% \mathrm{CV}$} & \multicolumn{2}{|c|}{$\%$ RE } & \multirow[t]{2}{*}{ Concentration } & \multicolumn{2}{|c|}{$\% \mathrm{CV}$} & \multicolumn{2}{|c|}{$\% \mathrm{RE}$} \\
\hline & & & Device 1 & Device 2 & Device 1 & Device 2 & & Device 1 & Device 2 & Device 1 & Device 2 \\
\hline A & Omeprazole & Low & 2.3 & 8.8 & 5.7 & 2.8 & High & 2.2 & 11.5 & 10.7 & 2.5 \\
\hline A & Midazolam & Low & 2.3 & 5.7 & 2.7 & -5.2 & High & 3.0 & 11.6 & 9.2 & -4.0 \\
\hline B & Cabazitaxel & Low & 7.7 & 5.3 & -12.7 & -15.0 & High & 5.5 & 2.3 & $-16.5^{\dagger}$ & -13.7 \\
\hline B & Fasiglifam & Low & 10.4 & 4.7 & 4.3 & 3.3 & High & 3.9 & 6.2 & 4.5 & -5.8 \\
\hline C & Trastuzumab & Low & 4.0 & 9.4 & -0.9 & -8.5 & High & 5.8 & 3.7 & 1.4 & -8.7 \\
\hline C & Eicosapentaenoic acid & Low & 13.3 & 8.1 & -9.4 & -3.1 & High & 9.7 & 4.3 & -4.7 & -6.9 \\
\hline D & Buprenorphine & Low & 2.4 & 2.3 & -8.4 & $-15.9 \dagger$ & High & 2.4 & 1.1 & -7.2 & $-21.1^{\dagger}$ \\
\hline D & Norbuprenorphine & Low & 1.4 & 2.2 & -3.9 & -0.9 & High & 1.5 & 2.1 & -4.1 & -10.2 \\
\hline D & Simvastatin & Low & 4.6 & 2.3 & -6.5 & -11.0 & High & 5.5 & 7.5 & -14.9 & $-19.4^{\dagger}$ \\
\hline $\mathrm{E}$ & Atenolol & Low & 4.3 & 7.3 & $-18.9^{\dagger}$ & -11.9 & High & 1.7 & 4.8 & $-19.7^{\dagger}$ & -4.0 \\
\hline$E$ & Warfarin & Low & 2.5 & 5.7 & -8.1 & -7.6 & High & 3.4 & 1.7 & -0.9 & -2.4 \\
\hline $\mathrm{F}$ & Clobazam & Low & $40.9^{\dagger}$ & 3.7 & 5.6 & 2.9 & High & $34.2^{\dagger}$ & 2.8 & $73.8^{\dagger}$ & -2.5 \\
\hline$F$ & Donepezil & Low & $38.6^{\dagger}$ & 3.3 & -4.2 & -8.0 & High & $32.6^{\dagger}$ & 6.1 & 14.4 & -10.4 \\
\hline G & Diclofenac & Low & $52.4^{\dagger}$ & 4.5 & $-52.9^{\dagger}$ & 4.6 & High & $16.2^{\dagger}$ & 6.6 & 4.0 & 7.3 \\
\hline G & Paracetamol & Low & $25.4^{\dagger}$ & 13.4 & 14.2 & $-21.7^{\dagger}$ & High & $21.0^{\dagger}$ & 6.5 & $42.6^{\dagger}$ & -8.0 \\
\hline
\end{tabular}

Table 2. Percentage of results within/outside each potential acceptance criteria.

\begin{tabular}{|c|c|c|c|c|}
\hline \multirow[t]{2}{*}{ Acceptance criteria } & \multicolumn{2}{|c|}{$\%$ CV from six replicates } & \multicolumn{2}{|c|}{$\%$ bias from control } \\
\hline & Device 1 & Device 2 & Device 1 & Device 2 \\
\hline$>15 \%$ & 27 & 0 & 20 & 13 \\
\hline$<15 \%$ & 73 & 100 & 80 & 87 \\
\hline$<10 \%$ & 67 & 90 & 63 & 67 \\
\hline$<5 \%$ & 50 & 50 & 37 & 37 \\
\hline
\end{tabular}

In general, the data look precise and accurate within our standard acceptance criteria of $15 \%$ with $73 \%$ or above of results being within $15 \%$. However, there are some outliers.

High \%CVs (>30\%) were generated for Clobazam, Donepezil, Diclofenac and Paracetamol using device 1, while the remainder of the $\% \mathrm{CV}$ for both devices are all $<15 \%$ with the majority of the values $<10 \%$. Two laboratories were involved in generating the data for Clobazam, Donepezil, Diclofenac and Paracetamol. These two laboratories had less experience using device 1 over the device 2 and, thus, struggled with the handling of the devices which may have led to this imprecision.

The accuracy data also show some outliers. $20 \%$ of results from device 1 and $13 \%$ from device 2 show a difference of $>15 \%$ from the controls. For the remainder of the data $(80 \%$ device 1 and $87 \%$ device 2$)$ it was seen that microsamples and traditional samples gave broadly equivalent results ( $<15 \%$ bias).

It was noted upon review of the data that the controls were not performed in the same way at every site; therefore, there may be some bias in the data due to the preparation of the controls.

Although the general trend of the data is precise and accurate, it also highlights the need for experience with the technique and a good understanding of the analytes. Where differences are observed, contributing factors may include preparation errors and lack of training. It is important to note that microsamples and conventional samples can give consistent results, provided adequate training with the technique and a good understanding of the analytes through development of the method. Naturally, the use of QC samples that mimic the study samples as closely as possible is key.

\section{Comparison of sample storage tubes}

In order to assess if the volume of storage tubes had any effect on the microsamples, 25-35 $\mu$ l of plasma from device 2 was dispensed into a tube with a capacity of $500 \mu \mathrm{l}$ (micrew tube) or $1100 \mu \mathrm{l}$ (micronic tube) and frozen prior to analysis. This assessment was performed by all contributing members for their specific analytes, using 


\begin{tabular}{|c|c|c|c|c|c|c|c|c|}
\hline Compound & Concentration & $8 \mu l$ & 1st $4 \mu \mathrm{I}$ & 2nd $4 \mu \mathrm{l}$ & Concentration & $8 \mu \mathrm{l}$ & 1st $4 \mu \mathrm{l}$ & 2nd $4 \mu \mathrm{l}$ \\
\hline Omeprazole & Low & 5.7 & 7.2 & 5.9 & High & 10.7 & 13.6 & 11.9 \\
\hline Midazolam & Low & 2.7 & 8.8 & 5.0 & High & 9.2 & 14.5 & 12.5 \\
\hline Cabazitaxel & Low & -12.7 & -11.7 & -0.8 & High & $-16.5^{\dagger}$ & -12.4 & -12.1 \\
\hline Fasiglifam & Low & 4.3 & -7.6 & -6.8 & High & 4.5 & -11.0 & -11.7 \\
\hline Eicosapentaenoic acid & Low & -9.4 & 8.2 & -9.4 & High & -4.7 & 11.3 & 1.7 \\
\hline Buprenorphine & Low & -8.4 & -10.6 & -10.4 & High & -7.2 & -6.5 & -8.1 \\
\hline Norbuprenorphine & Low & -3.9 & -6.4 & -6.3 & High & -4.1 & -2.1 & -3.9 \\
\hline Simvastatin & Low & -6.5 & $-68.2^{\dagger}$ & $-73.1^{\dagger}$ & High & -14.9 & $-50.0^{\dagger}$ & $-55.7^{\dagger}$ \\
\hline Warfarin & Low & -8.1 & -1.3 & -2.6 & High & -0.9 & 0.0 & 0.9 \\
\hline Clobazam & Low & 5.6 & $58.5^{\dagger}$ & $112.1^{\dagger}$ & High & $73.8^{\dagger}$ & $87.6^{\dagger}$ & $65.6^{\dagger}$ \\
\hline Donepezil & Low & -4.2 & $74.1^{\dagger}$ & $54.0^{\dagger}$ & High & 14.4 & $76.5^{\dagger}$ & $38.6^{\dagger}$ \\
\hline Diclofenac & Low & $-52.9^{\dagger}$ & $-45.8^{\dagger}$ & $-76.9^{\dagger}$ & High & 4.0 & $-33.5^{\dagger}$ & $-37.3^{\dagger}$ \\
\hline Paracetamol & Low & 14.2 & $-32.6^{\dagger}$ & $-16.1^{\dagger}$ & High & $42.6^{\dagger}$ & $-65.8^{\dagger}$ & $-82.4^{\dagger}$ \\
\hline
\end{tabular}

$\mathrm{n}=3-6$ replicates for each assessment. It was expected that small samples in the larger $1100 \mu \mathrm{l}$ may result in a loss of volume relative to the 500- $\mu$ l tube due to the effect of evaporative loss through a freeze-thaw cycle. Comparison of the data shows that $30 \%$ of the results from the $500-\mu \mathrm{l}$ tube compared with $13 \%$ of the results for the $1100-\mu \mathrm{l}$ tube had a bias from the control of greater than $15 \%$. This is the opposite to that expected and may have been due to mixing in the 500- $\mu$ l tubes as some of the contributing sites observed this to be more difficult in the smaller volume tubes. However, while in some cases, the data for the $500-\mu \mathrm{l}$ tube displayed a larger bias than the $1100-\mu \mathrm{l}$ tube the reverse is also present in the dataset. Therefore, there is no clear trend in the data.

Overall a similar number of results were seen to be acceptable for each tube type. 21 (70\%) and 26 (87\%; 500and $1100-\mu \mathrm{l}$ tubes, respectively) were within $15 \%$ of the control and $17(57 \%)$ and $20(67 \%)$ were within $10 \%$ of the control. No clear trends were observed during these investigations. It should be noted that the samples were stored for a relatively short period of time. It is possible that evaporative loss will be seen over a longer period and as a result stability should be assessed under the same conditions as the study samples will be stored.

\section{Homogeneity in device 1}

For device 1, the main area of investigation was the homogeneity of the plasma in the sampling capillary/capillaries. A total of $32 \mu \mathrm{l}$ of blood was collected into a capillary (Vitrex Micro Hematocrit Tube EDTA, Mark 32\&64) and after centrifugation either a single 8- $\mu$ l plasma sample or two 4- $\mu$ l plasma samples were taken up into accurate volume analytical capillaries (Vitrex End-to-End Micropipettes 4 and $8 \mu$ Plain). During sampling within a study it is possible for an $8-\mu \mathrm{l}$ capillary to be taken as a primary sample and a $4-\mu \mathrm{l}$ capillary as the secondary sample. The consortium sought to investigate the equivalence of these samples.

The protocol outlined preparation of plasma in either a single $8 \mu \mathrm{l}$ or $2 \times 4 \mu \mathrm{l}$ analytical capillaries. Consistent concentration results for each of the analytical capillaries would demonstrate homogeneity throughout the sampling capillary.

The data are presented in Table 3. In all cases, consistent results are obtained from both 4- $\mu$ l capillaries; therefore, there is clearly no difference in concentration of analyte along the capillary. However, in a number of cases (Simvastatin, Clobazam, Donepezil, Diclofenac and Paracetamol) the results for the 4- $\mu$ l capillaries differ from the 8- $\mu$ l capillaries. High CVs were obtained for Clobazam, Donepezil, Diclofenac and Paracetamol in both the 4- and 8- $\mu$ l capillaries using these devices. These experiments were carried out by two contributing sites and it is likely that the difference between the 4- and $8-\mu \mathrm{l}$ capillaries is in this case also due to insufficient experience with these devices. For Simvastatin, the data from the 8- $\mu$ l capillaries are precise and compare well to the control; however, the 4- $\mu$ l capillaries have a high degree of imprecision and inaccuracy compared with the controls. The reason for this is unclear but it does highlight the need to investigate subsampling during the method development 
and then the need for validating the 4 and $8 \mu$ samples as appropriate based on the intended sample collection and storage procedures.

\section{Point of dilution with device 1}

Typically, sample preparation protocols for device 1 use a dilution solvent added to a tube containing the accurate volume capillary either at the time of sample collection (in a study setting this is added by the those taking the blood from the animal or person and is therefore added prior to freezing) or on the first analysis occasion (in a study setting this is added by analytical scientists post thawing). Experiments were performed to investigate the impact of dilution at either point. Performing the dilution step before or after freezing of the sample has no impact on the results observed. In general, the precision data look better for the samples diluted prior to freezing, but in both cases the data are below $<10 \% \mathrm{CV}$ for the majority of analytes. It can be concluded that the precision and accuracy of the assay and the homogeneity of the samples are unlikely to be dependent on the point of dilution. It is recommended that each option should be considered carefully and experiments performed in the validation to accurately describe the sample collection method and subsequent storage conditions. Furthermore, the dilution step should be performed accurately and precisely by appropriately trained staff.

\section{Homogeneity in device 2}

With regards to device 2 , if any inhomogeneity was to be seen in the sampling it would be seen in the small volume of plasma dispensed into the sample tube after centrifugation of the device. Up to six replicates were taken from each of these samples and the results in Table 1 show that this device gave good precision data across the range of analytes tested; therefore, in the sample volumes tested (25-35 $\mu \mathrm{l})$ a homogeneous sample was obtained in all cases.

If any inhomogeneity was observed it is clear that a vortex mix of the samples prior to aliquotting was sufficient to overcome this.

\section{Point of dilution in device 2}

An additional investigation was performed to see if diluting the resultant plasma from device 2 prior to freezing would help overcome any homogeneity concerns by providing a larger sample volume for mixing. The results for the $\% \mathrm{CV}$ and the \%bias compared with the controls are inconclusive. In some cases, it appears that the dilution improves the precision and accuracy compared with the control but in the majority of cases there is no change or the precision and accuracy gets worse rather than better.

The dilution in this case was performed with the dilution solvent used for device 1 . As a consequence, the diluted samples contained less protein than the nondiluted samples. This may be the reason for the differences. It does however highlight the importance in selection of the dilution solvent for accurate quantitation and it is advised where possible to ensure the dilution solvent has properties as similar to plasma as possible. A dilution solvent that does not contain protein may be used as long as stability and adsorption is tested for and controlled appropriately.

\section{Conclusion}

The experimental results do not generate a clear set of conclusion. The data contain a lot of variability, which is contributed to by the broad range of experiences that each investigating site had with these techniques. The wide range of analytes with methods of varying sensitivity and complexity means that a number of factors could be contributing to every observation made.

It has been demonstrated that it is possible to generate homogeneous samples using these techniques and that dilution of microsamples can be of benefit if applied appropriately. It has also been demonstrated that the resultant microsamples can be accurate and precise. In addition, microsampling data have been demonstrated in many instances to be consistent with conventional larger volume plasma samples. If these targets cannot be achieved, then knowledge of the compounds, further experiments and experience with these techniques may help to overcome this.

With this in mind it may be sensible to take a cautious approach to implementation of microsampling techniques particularly when a company has no direct practical experience of using these techniques. Use of a well-established assay as a trial run and application within a discovery setting are both suitable ways to develop practical skills with the techniques as well as more knowledge and understanding of the typical complications. Owing to potential impacts on any sampling regime, consideration must also be given to the in-life facility during the study planning phase. 
For use in a regulatory environment, it had been seen that the techniques are capable of generating appropriate data but additional tests may need to be incorporated in the method development and validation. Where possible, homogeneity and stability should be assessed using the proposed sampling tubes and capillaries during the method development and/or validation. QC samples used to define the assay should be representative of the sample collection process.

\section{Future perspective}

Continued interest in microsampling techniques within the bioanalytical field is anticipated due to the increasing focus on the $3 R$ 's and the drive toward self-sampling within clinical applications. It is expected that a standard between companies will become evident in time. Until the point arrives where a standard is accepted and agreed, it is important that bioanalysts work in partnership with sample collection colleagues, to ensure that our validation and method development experiments are designed appropriately to ensure that QC samples are representative of the method of collecting and processing study samples.

\section{Executive summary}

\section{Background}

- The European Bioanalysis Forum has been engaging its members in high level technical discussions on the use and implementation on microsampling for some time.

- Two experimental protocols were prepared to assess: technical challenges in handling of small volumes (detailed in a separate paper) and perceived concerns of inhomogeneity relating to small volumes (this paper).

- The main experiments included in the protocol addressed the following questions:

- Are the results of liquid microsamples consistent with the respective conventional volume plasma samples?

- Does the volume of the sample tube selected for storage has any impact on the homogeneity of the samples?

- Is a plasma sample homogeneous within the capillary sampling device?

- If a dilution step is required to provide a viable sample at what point in the sampling or analytical process should this dilution be performed?

Results

- Results from the experiments performed are presented with regards to homogeneity, point of dilution, volume of storage tubes and comparison to macrosamples.

Conclusion

- Both techniques can produce homogeneous microsamples.

- Results from capillary microsamples can be generated to match macrosample values.

- However, the above conclusion is not always the case and a cautious approach to implementation of microsampling techniques in recommended.

\section{Acknowledgements}

The authors would like to thank all European Bioanalysis Forum members for very valuable input, especially the companies that performed the analytical work/generated the data - LGC, Lundbeck, Charles River, Sanofi, AstraZeneca, GlaxoSmithKline and Janssen R\&D.

Finally, special thanks to N Spooner for his contribution to the protocol planning stage during his time at GlaxoSmithKline.

\section{Disclaimer}

The views and conclusion presented in this paper are those of the European Bioanalysis Forum and do not necessarily reflect the representative affiliation or company's position on the subject.

Financial \& competing interests disclosure

The authors have no relevant affiliations or financial involvement with any organization or entity with a financial interest in or financial conflict with the subject matter or materials discussed in the manuscript. This includes employment, consultancies, honoraria, stock ownership or options, expert testimony, grants or patents received or pending, or royalties.

No writing assistance was utilized in the production of this manuscript. 


\section{Ethical conduct of research}

The authors state that they have obtained appropriate institutional review board approval or have followed the principles outlined in the Declaration of Helsinki for all human or animal experimental investigations. In addition, for investigations involving human subjects, informed consent has been obtained from the participants involved.

\section{References}

1. Timmerman P, White S, Cobb Z et al. Update of the EBF recommendation for the use of DBS in regulated bioanalysis integrating the conclusions from the EBF DBS-microsampling consortium. Bioanalysis 5(17), 2129-2136 (2013).

2. Timmerman P, White S, Cobb Z et al. European Bioanalysis Forum - continued plans to support liquid microsampling. Bioanalysis 6(14), 1897-1900 (2014).

3. White $\mathrm{S}$, Hawthorne G, Dillen L et al. European Bioanalysis Forum - reflection on bioanalytical assay requirements used to support Liquid Microsampling. Bioanalysis 6(19), 2581-2586 (2014).

4. Hawthorne G, Dillen L, Barfield $\mathrm{M}$ et al. Feedback from the EBF liquid micro sampling consortium: microsampling: assessing accuracy and precision of hand held pipettes and capillaries. Bioanalysis 11 (06), 533-542 (2019).

5. Cobb Z, de Vries R, Spooner N et al. In-depth study of homogeneity in DBS using two different techniques: results from the EBF DBS-micosampling consortium. Bioanalysis 5(17), 2161-2169 (2013). 